\title{
(Alpha, Beta)-Normal and Skew n-Normal Composite Multiplication Operator on Hilbert Spaces
}

\author{
Senthil $^{1,{ }^{*}, \text { Nithya }^{2}, \text { Suryadevi }^{3} \text {, David Chandrakumar }}{ }^{3}$ \\ ${ }^{1}$ Department of Economics and Statistics, Government of Tamilnadu, DRDA, Dindigul, India \\ ${ }^{2}$ Department of Mathematics, Mother Teresa Women's University, Kodaikanal, India \\ ${ }^{3}$ Department of Mathematics, Vickram College of Engineering, Enathi, India \\ Email address: \\ senthilsnc83@gmail.com (Senthil) \\ ${ }^{*}$ Corresponding author
}

To cite this article:

Senthil, Nithya, Suryadevi, David Chandrakumar. (Alpha, Beta)-Normal and Skew n-Normal Composite Multiplication Operator on Hilbert Spaces. International Journal of Discrete Mathematics. Vol. 4, No. 1, 2019, pp. 45-51. doi: 10.11648/j.dmath.20190401.17

Received: February 15, 2019; Accepted: March 19, 2019; Published: May 6, 2019

Abstract: In this paper, the condition under which composite multiplication operators on Hilbert spaces become skew nnormal operators, (Alpha, Beta)-normal, parahyponormal and quasi-parahyponormal have been obtained in terms of radonnikodym derivative.

Keywords: Composite Multiplication Operator, Conditional Expectation, Aluthge Transformation, Skew n-Normal Operator, Parahyponormal

\section{Introduction}

Let $\left(X, \sum, \mu\right)$ be a $\sigma$-finite measure space. Then a mapping $\mathrm{T}$ from $\mathrm{X}$ into $\mathrm{X}$ is said to be a measurable transformation if $T^{-1}(E) \in \sum$ for every $E \in \sum$. A measurable transformation $\mathrm{T}$ is said to be non-singular if $\mu\left(T^{-1}(E)\right)=0$ whenever $\mu(E)=0$. If $\mathrm{T}$ is non-singular then the measure $\mu T^{-1}$ defined as $\mu T^{-1}(E)=\mu\left(T^{-1}(E)\right)$ for every $\mathrm{E}$ in $\sum$, is an absolutely continuous measure on

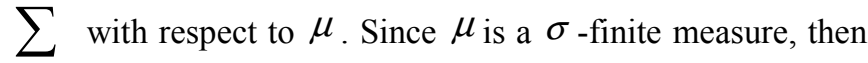
by the Radon-Nikodym theorem, there exists a non-negative function $f_{0}$ in $L^{1}(\mu)$ such that $\mu T^{-1}(E)=\int_{E} f_{0} d \mu$ for every $E \in \sum$. The function $f_{0}$ is called the RadonNikodym derivative of $\mu T^{-1}$ with respect to $\mu$.

Every non- singular measurable transformation $\mathrm{T}$ from $\mathrm{X}$ into itself induces a linear transformation $C_{T}$ on $L^{p}(\mu)$ defined as $C_{T} f=f \circ T$ for every $\mathrm{f}$ in $L^{p}(\mu)$. In case $C_{T}$ is continuous from $L^{p}(\mu)$ into itself, then it is called a composition operator on $L^{p}(\mu)$ induced by $\mathrm{T}$. We restrict our study of the composition operators on $L^{2}(\mu)$ which has Hilbert space structure. If $\mathrm{u}$ is an essentially bounded complexvalued measurable function on $\mathrm{X}$, then the mapping $M_{u}$ on $L^{2}(\mu)$ defined by $M_{u} f=u \cdot f$, is a continuous operator with range in $L^{2}(\mu)$. The operator $M_{u}$ is known as the multiplication operator induced by $u$. A composite multiplication operator is linear transformation acting on a set of complex valued $\sum$ measurable functions $f$ of the form

$$
M_{u, T}(f)=C_{T} M_{u}(f)=(u \circ T)(f \circ T)
$$

where $u$ is a complex valued, $\sum$ measurable function. In case $u=1$ almost everywhere, $M_{u, T}$ becomes a composition operator, denoted by $C_{T}$.

In the study considered is the using conditional expectation of composite multiplication operator on $L^{2}$-spaces. For eac $f \in L^{p}\left(X, \sum, \mu\right), 1 \leq p \leq \infty$, there exists an unique $T^{-1}\left(\sum\right)$-measurable function $E(f)$ such that 
$\int_{A} g f d \mu=\int_{A} g E(f) d \mu$

for every $T^{-1}\left(\sum\right)$-measurable function $g$, for which the left integral exists. The function $E(f)$ is called the conditional expectation of $f$ with respect to the subalgebra $T^{-1}\left(\sum\right)$. As an operator of $L^{p}(\mu), E$ is the projection onto the closure of range of $T$ and $E$ is the identity on $L^{p}(\mu), p \geq 1$ if and only if $T^{-1}\left(\sum\right)=\sum$. Detailed discussion of $E$ is found in [1-4].

\section{1. (Alpha, Beta)--Normal Operator [13]}

An operator $\mathrm{T}$ is called $(\alpha, \beta)$-normal operator if $\alpha^{2} T^{*} T \leq T T^{*} \leq \beta^{2} T^{*} T,(0 \leq \alpha \leq 1 \leq \beta)$

\subsection{Skew n-Normal Operator [12]}

An operator $\mathrm{T}$ is called skew n-normal operator if $\left(T^{n} T^{*}\right) T=T\left(T^{*} T^{n}\right)$, for all natural number $\mathrm{n}$.

\section{3. p-Hyponormal Operator [15]}

An operator $\mathrm{T}$ is called p-hyponormal operator if $\left(T^{*} T\right)^{p} \geq\left(T T^{*}\right)^{p}$, for $(0 \prec p \prec \infty)$

\section{Related Work in the Field}

The study of weighted composition operators on $L^{2}$ spaces was initiated by R. K. Singh and D. C. Kumar [5]. During the last thirty years, several authors have studied the properties of various classes of weighted composition operator. Boundedness of the composition operators in $L^{p}\left(\sum\right)$, $(1 \leq p<\infty)$ spaces, where the measure spaces are $\sigma$-finite, appeared already in [6]. Also boundedness of weighted operators on $C(X, E)$ has been studied in [7]. Recently $\mathrm{S}$. Senthil, P. Thangaraju and D. C. Kumar have proved several theorems on n-normal, n-quasi-normal, k-paranormal, and $(\mathrm{n}, \mathrm{k})$ paranormal of composite multiplication operators on $L^{2}$ spaces [8-11]. In this paper we investigate composite multiplication operators of $(\alpha, \beta)$-normal operator and skew n-normal operator $L^{2}(\mu)$-spaces.

\section{Hyponormality for Composite Multiplication Operator}

The results in the following proposition were proved in [12], as part of his doctoral dissertation.

\subsection{Proposition [3]}

Let $E=E(\cdot \backslash A)$ and let $\varphi$ be a non-negative $F$ measurable function.

Define the positive operator $P_{\varphi}$ by $P_{\varphi} f=\varphi E(\varphi f)$.

$$
\text { Let } \hat{\varphi}=\frac{\varphi}{\left(E\left(\varphi^{2}\right)\right)^{\frac{1}{4}}} \text {. Then } P_{\varphi}^{\frac{1}{2}}=P_{\hat{\varphi}} \text {. }
$$

Define the operator $R_{\varphi}$ by $R_{\varphi} f=E(\varphi f)$. Then $\left\|R_{\varphi}\right\|=\left\|\sqrt{E\left(\varphi^{2}\right)}\right\|_{\infty}$.

In [3], has proved the following lemma, as noted for any non-negative function $\mathrm{f}$,

$$
\text { sup port } f \subset \sup \text { port } E\left(f^{r}\right) \text { for any } r>0
$$

\subsection{Lemma [14]}

Let $\alpha$ and $\beta$ be non-negative functions, with $S=\sup$ port $\alpha$. Then the following are equivalent:

For every $f \in L^{2}(\mu) \int_{X} \alpha|f|^{2} d \mu \geq \int_{X}|E(\beta f \backslash A)|^{2} d \mu$ sup port $\beta \subset S$ and $E\left(\frac{\beta^{2}}{\alpha} \chi_{S} \backslash A\right) \leq 1$ almost everywhere.

\subsection{Proposition}

Let the composite multiplication operator $M_{u, T} \in B\left(L^{2}(\mu)\right)$. Then for $u \geq 0$

(i) $M_{u, T}^{*} M_{u, T} f=u^{2} f_{0} f$

(ii) $M_{u, T} M_{u, T}^{*} f=\left(u^{2} \circ T\right)\left(f_{0} \circ T\right) E(f)$

(iii) $M_{u, T}^{n}(f)=\left(C_{T} M_{u}\right)^{n}(f)=u_{n}\left(f \circ T^{n}\right), u_{n}=(u \circ T)\left(u \circ T^{2}\right)\left(u \circ T^{3}\right) \ldots \ldots \ldots\left(u \circ T^{n}\right)$

(iv) $M_{u, T}^{*} f=\left(u f_{0}\right)\left(E(f) \circ T^{-1}\right)$

(v) $M_{u, T}^{* n} f=u f_{0}\left(E\left(u f_{0}\right) \circ T^{-(n-1)}\right)\left(E(f) \circ T^{-n}\right)$

where $E\left(u f_{0}\right) \circ T^{-(n-1)}=\left(E\left(u f_{0}\right) \circ T^{-1}\right)\left(E\left(u f_{0}\right) \circ T^{-2}\right) \ldots \ldots .\left(E\left(u f_{0}\right) \circ T^{-(n-1)}\right)$

(vi) $\left|M_{u, T}\right|=u \sqrt{f_{0}} f$

(vii) $\left|M_{u, T}^{*}\right|=\left(\sqrt{u^{2} f_{0} \circ T}\right)\left(E\left(\sqrt{u^{2} f_{0} \circ T} f\right)\right)$

with the notation from Herron's proposition, 


$$
\left|M_{u, T}^{*}\right|=P_{\sqrt{u^{2} f_{0} \circ T}}=P_{v}, \text { where } v=\frac{\sqrt{\left(u^{2} f_{0}\right) \circ T}}{\left(E\left(\left(u^{2} f_{0}\right) \circ T\right)\right)^{\frac{1}{4}}}
$$

\section{Theorem 3.1}

Let the composite multiplication operator $M_{u, T} \in B\left(L^{2}(\mu)\right)$ with weight $u \geq 0$ and $\mathrm{S}$ be the support of $f_{0}$ :

$\left|M_{u, T}\right| \geq|C|$ if and only if $u^{2} \geq 1$

$|C| \geq\left|M_{u, T}^{*}\right|$ if and only if sup port $v=\sup$ port $\sqrt{f_{0}}$

where $v=\frac{\sqrt{\left(u^{2} f_{0}\right) \circ T}}{\left(E\left(\left(u^{2} f_{0}\right) \circ T\right)\right)^{\frac{1}{4}}}, E\left(\frac{\left(u^{2} f_{0}\right) \circ T /\left(E\left(u^{2} f_{0}\right) \circ T\right)^{\frac{1}{2}}}{\sqrt{f_{0}}} \chi_{S}\right) \leq 1$ almost everywhere.

Proof:

Since $\left|M_{u, T}\right|$ and $|C|$ are multiplication operators, we need only compare their symbols. After squaring, we get

$\left|M_{u, T}\right| \geq|C|$ if and only if $u \sqrt{f_{0}} \geq \sqrt{f_{0}}$

$\left|M_{u, T}\right| \geq|C|$ if and only if $u^{2} f_{0} \geq f_{0}$ Because $f_{0}>0$ almost everywhere,

we obtain (i).

As for this $\mathrm{f},|C| \geq\left|M^{*}{ }_{u, T}\right|$

$$
\begin{gathered}
\forall f, \int \sqrt{f_{0}}|f|^{2} d \mu \geq\left\langle\left|M_{u, T}^{*}\right| f, f\right\rangle \\
\left|M_{u, T}^{*}\right|=P_{v}, \text { where } v=\frac{\sqrt{\left(u^{2} f_{0}\right) \circ T}}{\left(E\left(\left(u^{2} f_{0}\right) \circ T\right)\right)^{\frac{1}{4}}}=\int v E(v f) \bar{f} d \mu
\end{gathered}
$$

However,

$$
\int v E(v f) \bar{f} d \mu=\langle E(v f), v f\rangle=\|E(v f)\|^{2}=\int|E(v f)|^{2} d \mu
$$

Since sup port $v=\sup$ port $\sqrt{f_{0}}$, the desired conclusion follows from lemma 3.2.

Theorem 3.5

Let the composite multiplication operator $M_{u, T} \in B\left(L^{2}(\mu)\right)$. Then $M_{u, T}$ is p-hyponormal if and only if $u f_{0}>0, u f_{0} \circ T>0$ and $E\left(1 / u^{2 p} f_{0}\right) \leq 1 / u^{2 p} f_{0} \circ T$.

Proof:

Here $\left\langle\left(M_{u, T}^{*} M_{u, T}\right)^{p} f, f\right\rangle=\int_{X} u^{2 p} f_{0}^{p}|f|^{2} d \mu$

and $\left\langle\left(M_{u, T} M_{u, T}^{*}\right)^{p} f, f\right\rangle=\int_{X}\left|E\left(\left(u^{p} f_{0}^{\frac{p}{2}}\right) \circ T f\right)\right|^{2} d \mu$

This implies $\int_{X} u^{2 p} f_{0}^{p}|f|^{2} d \mu \geq \int_{X}\left|E\left(\left(u^{p} f_{0}^{\frac{p}{2}}\right) \circ T f\right)\right|^{2} d \mu$

Since by lemma 2.2, for every $f \in L^{2}(\mu)$ 


$$
\begin{aligned}
& \Rightarrow \sigma\left(\left(u^{p} f_{0}^{\frac{p}{2}}\right) \circ T\right) \subset \sigma\left(u^{p} f_{0}^{p}\right) \text { and } E\left[\frac{\left(u^{2 p} f_{0}^{p}\right) \circ T}{u^{2 p} f_{0}^{p}}\right] \leq 1 \\
& \Rightarrow E\left[\frac{1}{u^{2 p} f_{0}^{p}}\right] \leq \frac{1}{\left(u^{2 p} f_{0}^{p}\right) \circ T} \text { if }\left(u^{2 p} f_{0}^{p}\right) \circ T>0 \text { and } u^{2 p} f_{0}^{p}>0 .
\end{aligned}
$$

\section{Parahyponormal for Composite Multiplication Operator}

Mahmound M. Kutkut [16] , has proved that an operator A is parahyponormal if and only if $\left(A A^{*}\right)^{2}-2 C A^{*} A+C^{2} \geq 0$ for all real C. In an analogous manner, we derive some characterization of parahyponormal and quasi-parahyponormal composite multiplication operator on $L^{2}$-spaces.

Theorem 4.1

Let the composite multiplication operator $M_{u, T} \in B\left(L^{2}(\mu)\right)$. Then $M_{u, T}$ is parahyponormal if and only if $u^{4} \circ T \cdot f_{0}^{2} \circ T E(f)-2 C u^{2} f_{0} f+C^{2} \geq 0$ almost everywhere, for all $C \geq 0$

Proof:

Suppose $M_{u, T}$ is parahyponormal. Then $\left(M_{u, T} M_{u, T}^{*}\right)^{2}-2 C M_{u, T}^{*} M_{u, T}+C^{2} \geq 0$ for all $C \geq 0$.

This implies that

$$
\left\langle\left(\left(M_{u, T} M^{*}{ }_{u, T}\right)^{2}-2 C M_{u, T}^{*} M_{u, T}+C^{2}\right) f, f\right\rangle \geq 0 \text { for all } f \in L^{2}(\mu)
$$

By the proposition 3.3 we get,

$$
\begin{aligned}
& \int_{E}\left\{\left(u^{2} \circ T \cdot f_{0} \circ T \cdot E(f)\right)^{2}-2 C u^{2} f_{0} f+C^{2}\right\} d \mu \geq 0 \text { for every } E \in \sum . \\
& \Leftrightarrow u^{4} \circ T \cdot f_{0}^{2} \circ T E(f)-2 C u^{2} f_{0} f+C^{2} \geq 0 \text { almost everywhere, for all } C \geq 0
\end{aligned}
$$

Theorem 4.2

Let the composite multiplication operator $M_{u, T} \in B\left(L^{2}(\mu)\right)$. Then $M_{u, T}$ is M-parahyponormal if and only if $m^{2} u^{4} \circ T \cdot f_{0}^{2} \circ T E(f)-2 C u^{2} f_{0} f+C^{2} \geq 0$ almost everywhere, for all $C \geq 0$.

Proof:

Suppose $M_{u, T}$ is M-parahyponormal.

Then $m^{2}\left(M_{u, T} M_{u, T}^{*}\right)^{2}-2 C M_{u, T}^{*} M_{u, T}+C^{2} \geq 0$ for all $C \geq 0$.

This implies that

$$
\left\langle\left(m^{2}\left(M_{u, T} M_{u, T}^{*}\right)^{2}-2 C M_{u, T}^{*} M_{u, T}+C^{2}\right) f, f\right\rangle \geq 0 \text { for all } f \in L^{2}(\mu)
$$

By the proposition 3.3 we get,

$$
\begin{aligned}
& \int_{E}\left\{m^{2}\left(u^{2} \circ T \cdot f_{0} \circ T \cdot E(f)\right)^{2}-2 C u^{2} f_{0} f+C^{2}\right\} d \mu \geq 0 \text { for every } E \in \sum . \\
& \Leftrightarrow m^{2} u^{4} \circ T \cdot f_{0}^{2} \circ T E(f)-2 C u^{2} f_{0} f+C^{2} \geq 0 \text { almost everywhere, for all } C \geq 0
\end{aligned}
$$

Theorem 4.3

Let the composite multiplication operator $M_{u, T} \in B\left(L^{2}(\mu)\right)$. Then $M_{u, T}$ is $M^{*}$-parahyponormal if and only if $m^{2} u^{2} f_{0} E\left(u^{2} f_{0}\right) \circ T^{-1} f-2 C u^{2} \circ T \cdot f_{0} \circ T E(f)+C^{2} \geq 0$ almost everywhere, for all $C \geq 0$.

Proof:

Suppose $M_{u, T}$ is $M^{*}$-parahyponormal.

Then $m^{2} M_{u, T}^{*^{2}} M_{u, T}^{2}-2 C M_{u, T} M_{u, T}^{*}+C^{2} \geq 0$ for all $C \geq 0$.

This implies that

$$
\left\langle\left(m^{2} M^{*^{2}}{ }_{u, T} M_{u, T}^{2}-2 C M_{u, T} M^{*}{ }_{u, T}+C^{2}\right) f, f\right\rangle \geq 0 \text { for all } f \in L^{2}(\mu)
$$

By the proposition 3.3 and $M^{*^{2}}{ }_{u, T} M^{2}{ }_{u, T} f=u^{2} f_{0} E\left(u^{2} f_{0}\right) \circ T^{-1} f u \geq 0$ we get,

$$
\int_{E}\left\{m^{2} u^{2} f_{0} E\left(u^{2} f_{0}\right) \circ T^{-1} f-2 C u^{2} \circ T \cdot f_{0} \circ T E(f)+C^{2}\right\} d \mu \geq 0 \text { for every } E \in \sum \text {. }
$$


$\Leftrightarrow m^{2} u^{2} f_{0} E\left(u^{2} f_{0}\right) \circ T^{-1} f-2 C u^{2} \circ T \cdot f_{0} \circ T E(f)+C^{2} \geq 0$ almost everywhere, for all $C \geq 0$

\section{Quasi-Parahyponormal for Composite Multiplication Operator}

By result of [16] an operators $\mathrm{A}$ on $\mathrm{H}$ is quasi-parahyponormal if and only if $\left(A^{2} A^{*^{2}}\right)^{2}-2 C\left(A A^{*}\right)^{2}+C^{2} \geq 0$ for all C. In an analogous manner, we derive the characterization of quasi-parahyponormal composite multiplication operator on $L^{2}$-spaces.

Theorem 5.1

Let the composite multiplication operator $M_{u, T} \in B\left(L^{2}(\mu)\right)$. Then $M_{u, T}$ is quasi-parahyponormal if and only if $u^{2} \circ T \cdot u^{4} \circ T^{2} \cdot f_{0}^{2} \circ T^{2} \cdot\left(E\left(u f_{0}\right)\right)^{2} \circ T \cdot E(f)-2 C u^{4} \circ T \cdot f_{0}^{2} \circ T \cdot E(f)+C^{2} \geq 0$ almost everywhere, for all $C \geq 0$

Proof:

Suppose $M_{u, T}$ is quasi-parahyponormal.

Then $\left(M_{u, T}^{2} M^{*^{2}}{ }_{u, T}\right)^{2}-2 C\left(M_{u, T} M^{*}{ }_{u, T}\right)^{2}+C^{2} \geq 0$ for all $C \geq 0$.

This implies that

$\left\langle\left(\left(M_{u, T}^{2} M^{*^{2}}{ }_{u, T}\right)^{2}-2 C\left(M_{u, T} M^{*}{ }_{u, T}\right)^{2}+C^{2}\right) f, f\right\rangle \geq 0$ for all $f \in L^{2}(\mu)$

By the proposition 3.3 and $M_{u, T}^{2} M^{*^{2}}{ }_{u, T} f=u \circ T \cdot u^{2} \circ T^{2} \cdot f_{0} \circ T^{2} \cdot E\left(u f_{0}\right) \circ T \cdot E(f)$

$\int_{E}\left\{\left(u \circ T \cdot u^{2} \circ T^{2} \cdot f_{0} \circ T^{2} \cdot E\left(u f_{0}\right) \circ T \cdot E(f)\right)^{2}-2 C\left(u^{2} \circ T \cdot f_{0} \circ T \cdot E(f)\right)^{2}+C^{2}\right\} d \mu \geq 0$ for every $E \in \sum$

$\Leftrightarrow u^{2} \circ T \cdot u^{4} \circ T^{2} \cdot f_{0}^{2} \circ T^{2} \cdot\left(E\left(u f_{0}\right)\right)^{2} \circ T \cdot E(f)-2 C u^{4} \circ T \cdot f_{0}^{2} \circ T \cdot E(f)+C^{2} \geq 0$

almost everywhere, for all $C \geq 0$

Theorem 5.2

Let the composite multiplication operator $M_{u, T} \in B\left(L^{2}(\mu)\right)$. Then $M_{u, T}$ is M-quasi-parahyponormal if and only if $m^{2} u^{2} \circ T \cdot u^{4} \circ T^{2} \cdot f_{0}^{2} \circ T^{2} \cdot\left(E\left(u f_{0}\right)\right)^{2} \circ T \cdot E(f)-2 C u^{4} \circ T \cdot f_{0}^{2} \circ T \cdot E(f)+C^{2} \geq 0$ almost everywhere, for all $C \geq 0$

Proof:

Suppose $M_{u, T}$ is M-quasi-parahyponormal.

Then $m^{2}\left(M_{u, T}^{2} M_{u, T}^{*^{2}}\right)^{2}-2 C\left(M_{u, T} M^{*}{ }_{u, T}\right)^{2}+C^{2} \geq 0$ for all $C \geq 0$.

This implies that

$\left\langle\left(m^{2}\left(M_{u, T}^{2} M^{*^{2}}{ }_{u, T}\right)^{2}-2 C\left(M_{u, T} M^{*}{ }_{u, T}\right)^{2}+C^{2}\right) f, f\right\rangle \geq 0$ for all $f \in L^{2}(\mu)$

By the proposition 3.3 and $M_{u, T}^{2} M^{*^{2}}{ }_{u, T} f=u \circ T \cdot u^{2} \circ T^{2} \cdot f_{0} \circ T^{2} \cdot E\left(u f_{0}\right) \circ T \cdot E(f)$

$\int_{E}\left\{m^{2}\left(u \circ T \cdot u^{2} \circ T^{2} \cdot f_{0} \circ T^{2} \cdot E\left(u f_{0}\right) \circ T \cdot E(f)\right)^{2}-2 C\left(u^{2} \circ T \cdot f_{0} \circ T \cdot E(f)\right)^{2}+C^{2}\right\} d \mu \geq 0$ for every $E \in \sum$.

$\Leftrightarrow m^{2} u^{2} \circ T \cdot u^{4} \circ T^{2} \cdot f_{0}^{2} \circ T^{2} \cdot\left(E\left(u f_{0}\right)\right)^{2} \circ T \cdot E(f)-2 C u^{4} \circ T \cdot f_{0}^{2} \circ T \cdot E(f)+C^{2} \geq 0$ almost everywhere, for all $C \geq 0$

\section{Skew n-normal and (Alpha, Beta)-normal Composite Multiplication Operator}

Theorem 6.1

Let the composite multiplication operator $M_{u, T} \in B\left(L^{2}(\mu)\right)$. Then $M_{u, T}$ is skew n-normal if and only if $u_{n}\left(u^{2} \circ T^{n}\right)\left(f_{0} \circ T^{n}\right)=\left(u^{2} \circ T\right)\left(f_{0} \circ T\right)\left(E\left(u_{n}\right) \circ T\right)$ almost everywhere.

Proof:

$M_{u, T}$ is skew n-normal. Then

$$
\begin{gathered}
\left(M_{u, T}^{n} M_{u, T}^{*}\right) M_{u, T} f=\left(M_{u, T}^{n} M_{u, T}^{*}\right)(u f) \circ T \\
=M_{u, T}^{n}\left(u f_{0} E((u f) \circ T) \circ T^{-1}\right)
\end{gathered}
$$




$$
\begin{gathered}
=M_{u, T}^{n} u^{2} f_{0} f \\
=u_{n}\left(u^{2} f_{0} f\right) \circ T^{n} \\
=u_{n}\left(u^{2} \circ T^{n}\right)\left(f_{0} \circ T^{n}\right)\left(f \circ T^{n}\right)
\end{gathered}
$$

Also,

$$
\begin{aligned}
M_{u, T}\left(M_{u, T}^{*} M_{u, T}^{n}\right) f & =M_{u, T} M_{u, T}^{*}\left(u_{n}\left(f \circ T^{n}\right)\right) \\
= & M_{u, T} u f_{0} E\left(u_{n}\left(f \circ T^{n}\right)\right) \circ T^{-1} \\
= & M_{u, T} u f_{0} E\left(u_{n}\right)\left(f \circ T^{n-1}\right) \\
= & u\left(u f_{0} E\left(u_{n}\right)\left(f \circ T^{n-1}\right)\right) \circ T \\
= & \left(u^{2} \circ T\right)\left(f_{0} \circ T\right) E\left(u_{n}\right) \circ T\left(f \circ T^{n}\right)
\end{aligned}
$$

$\Leftrightarrow u_{n}\left(u^{2} \circ T^{n}\right)\left(f_{0} \circ T^{n}\right)=\left(u^{2} \circ T\right)\left(f_{0} \circ T\right)\left(E\left(u_{n}\right) \circ T\right)$ almost everywhere.

Theorem 6.2

Let the composite multiplication operator $M_{u, T} \in B\left(L^{2}(\mu)\right)$. Then $M^{*}{ }_{u, T}$ is skew n-normal if and only if $u^{2} f_{0}^{2}=\left(u^{2} \circ T^{-(n-1)}\right)\left(f_{0} \circ T^{-(n-1)}\right)$ almost everywhere.

Proof:

$M^{*}{ }_{u, T}$ is skew n-normal. Then

$$
\begin{gathered}
M_{u, T}^{*}\left(M_{u, T} M^{* n}{ }_{u, T}\right) f=M^{*}{ }_{u, T} M_{u, T}\left(u f_{0}\left(E\left(u f_{0}\right) \circ T^{-(n-1)}\right)\left(E(f) \circ T^{-n}\right)\right) \\
=M^{*}{ }_{u, T}\left(u\left(u f_{0}\left(E\left(u f_{0}\right) \circ T^{-(n-1)}\right)\left(E(f) \circ T^{-n}\right)\right)\right) \circ T \\
=M^{*}{ }_{u, T}\left(\left(u^{2} \circ T\right)\left(f_{0} \circ T\right)\left(E\left(u f_{0}\right) \circ T^{-(n+2)}\right)\left(E(f) \circ T^{-(n-1)}\right)\right) \\
=u f_{0} E\left[\left(\left(u^{2} \circ T\right)\left(f_{0} \circ T\right)\left(E\left(u f_{0}\right) \circ T^{-(n+2)}\right)\left(E(f) \circ T^{-(n-1)}\right)\right)\right] \circ T^{-1} \\
=u^{3} f_{0}^{2}\left(E\left(u f_{0}\right) \circ T^{-(n-1)}\right)\left(E(f) \circ T^{-n}\right)
\end{gathered}
$$

Also,

$$
\begin{gathered}
\left(M_{u, T}^{* n} M_{u, T}\right) M_{u, T}^{*} f=M_{u, T}^{* n} M_{u, T}\left(u f_{0} E(f) \circ T^{-1}\right) \\
=M_{u, T}^{* n}\left(\left(u^{2} \circ T\right)\left(f_{0} \circ T\right) E(f)\right) \\
=u f_{0} E\left(u f_{0}\right) \circ T^{-(n-1)}\left(u^{2} \circ T^{-(n-1)}\right)\left(f_{0} \circ T^{-(n-1)}\right) E(f) \circ T^{-n}
\end{gathered}
$$

$\Leftrightarrow u^{2} f_{0}^{2}=\left(u^{2} \circ T^{-(n-1)}\right)\left(f_{0} \circ T^{-(n-1)}\right)$ almost everywhere.

Theorem 6.2

Let the composite multiplication operator $M_{u, T} \in B\left(L^{2}(\mu)\right)$. Then $M_{u, T}$ is $(\alpha, \beta)$-normal if and only if $\alpha^{2} u^{2} f_{0} f \leq\left(u^{2} f_{0}\right) \circ T E(f) \leq \beta^{2} u^{2} f_{0} f$ almost everywhere.

Proof:

$M_{u, T}$ is $(\alpha, \beta)$-normal. Thenit easy to check, 


$$
M_{u, T}^{*} M_{u, T} f=u^{2} f_{0} f, M_{u, T} M_{u, T}^{*} f=\left(u^{2} \circ T\right)\left(f_{0} \circ T\right) E(f)
$$

By definition,

$$
\alpha^{2} u^{2} f_{0} f \leq\left(u^{2} f_{0}\right) \circ T E(f) \leq \beta^{2} u^{2} f_{0} f \text { almost everywhere. }
$$

\section{Conclusion}

In the study of p-hyponormal operator, the Aluthge transform is a very useful tool. We investigate some basic properties of such operators and study the relation among skew n-normal operators, $(\alpha, \beta)$-normal operator, parahyponormal and quasi-parahyponormal composite multiplication operators on $L^{2}(\mu)$-space. In future try to generalize the composite multiplication operator on Poisson weighted sequence spaces.

\section{Acknowledgements}

We would like to thank the reviewers for carefully reading manuscript and for their constructive comments. I want to thank Professor Dr. R. David Chandrakumar, Department of Mathematics, Vickram College of Engineering for his support and encouragement during preparation of the paper.

\section{References}

[1] Campbell J \& Jamison J, On some classes of weighted composition operators, Glasgow Math. J. vol. 32, pp. 82-94, (1990).

[2] Embry Wardrop M \& Lambert A, Measurable transformations and centred composition operators, Proc. Royal Irish Acad, vol. 2(1), pp. 23-25 (2009).

[3] Herron J, Weighted conditional expectation operators on Hilbert spaces, UNC charlotte doctoral dissertation.

[4] Thomas Hoover, Alan Lambert and Joseph Quinn, The Markov process determined by a weighted composition operator, Studia Mathematica, vol. XXII (1982).

[5] Singh RK \& Kumar DC, Weighted composition operators, Ph.D. thesis, Univ. of Jammu (1985).
[6] Singh RK, Composition operators induced by rational functions, Proc. Amer. Math. Soc., vol. 59, pp. 329-333(1976).

[7] Takagi H \& Yokouchi K, Multiplication and Composition operators between two Hilbert spaces, Contem. Math., vol. 232, pp. 321-338 (1999).

[8] PanaiyappanS \& Senthil kumar D, Parahyponormal and Mparahyponormal composition operators, Acta Ciencia Indica, vol. XXVIII (4) (2002).

[9] Senthil S, Thangaraju P \& Kumar DC, n-normal and n-quasinormal composite multiplication operator on Hilbert spaces, Journal of Scientific Research \& Reports, vol. 8(4), pp. 1-9 (2015).

[10] Senthil S, Thangaraju P \& KumarDC, k-*paranormal, k-quasi*paranormal and (n,k)- quasi-*paranormal composite multiplication operator on Hilbert spaces, British Journal of mathematics and computer science, BJMCS20166 (2015).

[11] Senthil S, Thangaraju P \& Kumar DC, Composite multiplication operator on Hilbert spaces of vector valued functions, International research Journal of Mathematical Sciences, vol. 4 (2), pp. 1(2015).

[12] Shaakir LK\& Abdulwahid ES, Skew n-normal operators, Aust. J. of basic and appl. sci, Vol-8(16), pp. 340-344 (2014).

[13] Moslehian MS, On (Alpha, Beta)-normal operators in Hilbert spaces, IMAGE 39, Problem 39-4 (2007).

[14] Burnap C, Jung I \&Lambert A, Separating partial normality classes with composition operators, J. Operator Theory Vol. 53(2), pp. 381-397 (2005).

[15] Fujii M \& Nakatsu Y, On subclasses of hyponormal operators, Proc. Japan Acad. Ser. A. Math. Sci. vol. (51), pp. 243-246 (1975).

[16] Kutkut \& Mahmoud M, On the class of parahyponormal operators, J. Math Sci, (Calcutta), vol. 4(2), pp. 73-88 (1993). 